\title{
PÓS-COLHEITA DE FOLHAS DE ESPÉCIES DE Strelitizia spp
}

Jean Carlo Baudraz de Paula ${ }^{1 *}$, Walter Aparecido Ribeiro Júnior ${ }^{1}$, Gabriel Danilo Shimizu ${ }^{1}$, Gabriel Barraca Men², Camilla de Andrade Pacheco ${ }^{3}$, Ricardo Tadeu de Faria ${ }^{4}$

\footnotetext{
1 Doutorando, Agronomia, Universidade Estadual de Londrina (UEL), Londrina, PR *E-mail do autor correspondente: jc_baudraz@live.com

${ }^{2}$ Graduando, Agronomia, Universidade Estadual de Londrina (UEL), Londrina, PR.

${ }^{3}$ Pesquisadora e Pós-doutoranda na área de Epidemiologia do Fundo de Defesa da Citricultura (Fundecitrus), Araraquara, SP.

${ }^{4}$ Docente, Fitotecnia, Universidade Estadual de Londrina (UEL), Londrina, PR.
}

\section{Recebido: 20/03/2020; Aceito: 10/12/2020}

RESUMO: A floricultura constitui um dos segmentos mais dinâmicos do agronegócio e a utilização de arranjos florais exige alto padrão de qualidade de seus produtos e uma maior longevidade pós-colheita das flores. A quitosana é um polissacarídeo biodegradável capaz de formar um filme natural e permeável às trocas gasosas realizadas pelo vegetal, permitindo a redução do metabolismo da planta favorecendo o prolongamento de sua vida útil. Este estudo teve como objetivo avaliar a durabilidade pós-colheita de folhas de Strelitizia reginae e Strelitizia juncea utilizando produto comercial a base de quitosana (FishFertil $\left.{ }^{\circledR}\right)$. O experimento foi conduzido inteiramente casualisado de 6 tratamentos com 5 repetições (onde cada folha foi considerada como uma repetição). Foram utilizadas 6 doses $\left(0 ; 10 ; 20 ; 40 ; 80\right.$ e $160 \mathrm{mg} \mathrm{L}^{-1}$ do produto comercial). Realizaram-se avaliações visuais da longevidade das folhas através de notas e o acompanhamento da perda de água nos tecidos foliares. Observou-se que FishFertil® retardou a perda de massa fresca das folhas de $S$. juncea e $S$. reginae. Para ambas as espécies o tratamento controle permaneceu até o $22^{\circ}$ dia com notas aceitáveis comercialmente (nota 4). $\mathrm{O}$ produto retardou a perda de massa fresca das folhas de $S$. juncea e $S$. reginae e as mantiveram até o $34^{\circ}$ dia notas dentro do padrão comercial, contudo não foi possível determinar a dose de máxima eficiência do produto para as duas espécies de Strelitzia.

Palavras-chave: Conservação. Fishfertil®. Quitosana. Strelitzia juncea. Strelitzia reginae.

\section{POSTHARVEST LEAVES OF SPECIES OF Strelitizia spp.}

\begin{abstract}
Floriculture is one of the most dynamic segments of agribusiness and the use of floral arrangements requires high quality standards of its products and greater post-harvest longevity of flowers. Chitosan is a biodegradable polysaccharide capable of forming a natural and permeable film to the gas exchange carried out by the plant, allowing the reduction of the plant's metabolism favoring the prolongation of its useful life. This study aimed to evaluate the postharvest durability of Strelitizia reginae and Strelitizia juncea leaves using a commercial product based on chitosan (FishFertil®). The experiment was conducted entirely randomized with 6 treatments with 5 repetitions (where each leaf was considered as one repetition). Six doses were used $\left(0 ; 10 ; 20 ; 40 ; 80\right.$ and $160 \mathrm{mg} \mathrm{L}^{-1}$ of the commercial product). Visual assessments of leaf longevity were carried out through notes and the monitoring of water loss
\end{abstract}


in leaf tissues. It was observed that FishFertil® delayed the loss of fresh weight of the leaves of $S$. juncea and S. reginae. For both species, the control treatment remained until the 22nd day with commercially acceptable grades (grade 4). The product delayed the loss of fresh mass of the leaves of $S$. juncea and $S$. reginae and kept them up to the 34th day notes within the commercial standard, however it was not possible to determine the maximum efficiency dose of the product for the two species of Strelitzia.

Key words: Conservation. Fishfertil@. Chitosan. Strelitzia juncea. Strelitzia reginae.

\section{INTRODUÇÃO}

A floricultura abrange o cultivo de flores e plantas ornamentais com variados fins, constituindo um dos segmentos mais dinâmicos do agronegócio brasileiro contemporâneo, tornando-se uma atividade atrativa aos produtores (NEVES; PINTO, 2015). Dados do Instituto Brasileiro de Floricultura (IBRAFLOR) comprovam o aumento deste mercado, onde faturamento para o setor foi de R \$ 8,7 bilhões em 2019, representando um aumento de 7\% em relação a 2018. Um fator que tem impulsionado esse aumento é a maior disponibilidade de renda da população e a facilidade de compra, através do desenvolvimento de novos canais utilizados na comercialização, como supermercados e vendas online (IBRAFLOR, 2019).

No comércio de ornamentais existe uma procura muito grande por folhagens e complementos para atender o setor de flores cortadas (NEVES; PINTO, 2015). A Strelitzia, popularmente conhecida como "ave-do-paraíso" (VIEIRA et al., 2012), é uma planta herbácea, perene, originária da África do Sul, de 1,2 a 1,5 m de altura, de florescimento decorativo, com folhas firmes e coriáceas (LORENZI; SOUZA, 2008). Sua comercialização como planta de corte é advinda à grande durabilidade pós-colheita de suas plantas (PIVETTA; BARBOSA; ARAÚJO, 2007).

Para que a comercialização seja bem sucedida, é importante a manutenção da qualidade pós-colheita, de modo que todo o esforço empregado durante a produção seja compensado no momento da venda do produto. Neste cenário, destaca-se o manejo pós-colheita, por reduzir os processos metabólicos, principalmente taxa respiratória, produção de etileno e transpiração (DIAS-TAGLIACOZZO; MOSCA, 2007).

Os fatores que mais contribuem para a diminuição da qualidade pós-colheita da ave-doparaíso são a deterioração e perda de brilho dos tecidos e órgãos, além de manchas e perda da coloração dos mesmos (MACNISH et al., 2009). Não obstante, para se conquistar novos mercados, há a necessidade da adoção de técnicas de conservação pós-colheita que possibilitem melhorias na qualidade da Strelitzia (AUSTRALIA, 2019).

Um dos produtos utilizados que permite maior longevidade e resistência a patógenos póscolheita é a quitosana, um amino polissacarídeo derivado da desacetilação da quitina, composto orgânico de propriedades solventes e umectantes, capaz de formar um filme natural e permeável às trocas gasosas realizadas pelo vegetal, permitindo a redução do metabolismo da planta e, consequentemente, o prolongamento de sua vida útil (LARANJEIRA; FÁVERE, 2009). 
Considerando o elevado potencial ornamental de folhagens de Strelitzia, aliada a escassos estudos sobre pós-colheita e a baixa utilização de quitosana no mercado de flores, o objetivo deste trabalho foi avaliar a longevidade pós-colheita de folhas de $S$. reginae e $S$. juncea utilizando o produto comercial a base de quitosana FishFertil ${ }^{\circledR}$.

\section{MATERIAL E MÉTODOS}

O experimento foi realizado no Laboratório de Fitotecnia/Departamento de Agronomia/Universidade Estadual de Londrina, entre 19 de Abril a 29 de Maio de 2018. As folhas foram colhidas de um lote de plantas da referida universidade, no período da manhã $(8 \mathrm{~h} 00$ às $9 \mathrm{~h} 00)$.

As folhas presentes nas plantas de ambas as espécies foram coletadas no estádio juvenil, localizadas no terço inferior (base) e das plantas, previamente selecionadas, descartando-se aquelas com defeitos físicos, fisiológicos e/ou fitopatogênicos. Posteriormente as mesmas foram padronizadas quanto ao tamanho e peso: Para Strelitizia reginae $40 \pm 2 \mathrm{~cm}$ de comprimento, $10,5 \pm 0,5 \mathrm{~cm}$ de largura e peso médio de 39,6 $\pm 5 \mathrm{~g}$ já Strelitizia juncea $23 \pm 2 \mathrm{~cm}$ de comprimento, $3.8 \pm 0,5 \mathrm{~cm}$ de largura e peso médio de $18,6 \pm 5 \mathrm{~g}$.

As folhas foram previamente lavadas em água corrente, para remoção de impurezas superficiais, e posteriormente cortadas em bisel dentro de um recipiente plástico $(29 \times 29 \times 30 \mathrm{~cm})$ contendo água potável, com o auxílio de uma tesoura de poda, obtendo-se uma haste de $30 \mathrm{~cm}$ de comprimento.

O conservante utilizado foi o FishFertil ${ }^{\circledR}$ (concentração de quitosana de $20 \mathrm{~g} \mathrm{~L}^{-1}$ ), pesado em miligrama $(\mathrm{mg})$ em uma balança semianalítica $( \pm 0,01 \mathrm{~g})$, e feita a diluição do mesmo, para cada tratamento, em 1L de água destilada. Os tratamentos realizados foram: água destilada, 10, 20, 40, 80 e $160 \mathrm{mg} \mathrm{L}^{-1}$. Em seguida, as folhas foram imersas em recipientes plásticos $(29 \times 29 \times 30 \mathrm{~cm})$ contento a quantidade descrita para cada tratamento de solução conservante e permaneceram submersas durante 15 segundos.

Após esse tempo, as folhas foram colocadas em recipientes de vidro com capacidade de $200 \mathrm{~mL}$ contendo água destilada, onde permaneceram durante todo o experimento. Foi acrescido $5 \mathrm{~mL}$ de hipoclorito de sódio a $3 \%$ como agente bactericida, sendo esta solução renovada a cada três dias. As folhas permaneceram em local com temperatura mantida a $22 \pm 2^{\circ} \mathrm{C}$, com $60 \%$ de umidade relativa e $2000 /$ lux de luminosidade.

As avaliações visuais da longevidade das folhas, considerando os atributos de cor, brilho e turgidez, foram realizadas a cada dois dias, durante 39 dias (Figuras 1 e 2), segundo o critério de notas adaptado (DIAS-TAGLIACOZZO; CASTRO, 2005; GUIMARÃES 2008; VIEIRA et al., 2013): Nota 4 (excelente): folhas túrgidas com brilho e coloração; Nota 3 (boa): folhas com amarelecimento e/ou murchas e pequena perda de brilho nas folhas; Nota 2 (regular): folhas com ressecamento nas extremidades, sintomas de murcha, perda de cor e brilho; Nota 1 (ruim): ressecamento evidenciado pela perda de brilho e turgência, murcha avançada e perda de cor; Nota 0 (descarte): folhas moles, sem turgidez, sem brilho e com ressecamento total ou escurecimento. 


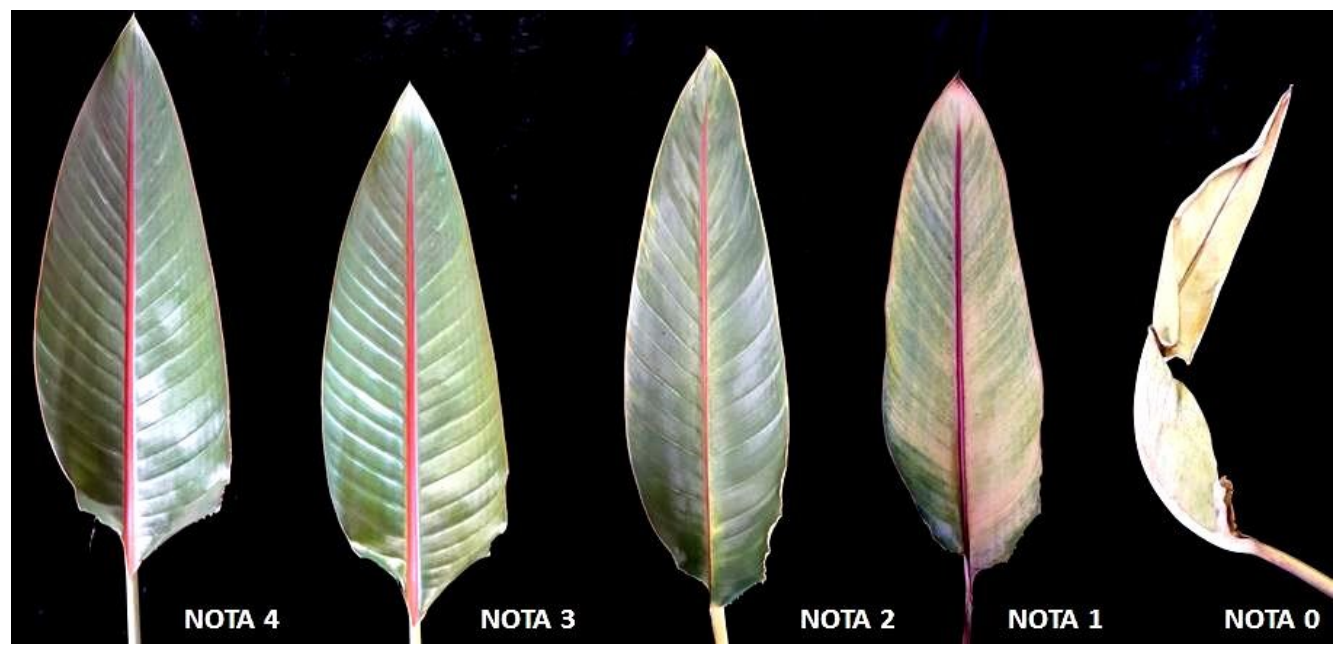

Figura 1. Critério de classificação por notas para avaliação da longevidade pó-colheita das folhas de Strelitzia reginae. Londrina/PR, 2018. Grading criteria for grades to assess postharvest longevity of Strelitzia reginae leaves. Londrina/PR, 2018.

Fonte: Autoria própria. Own authorship.

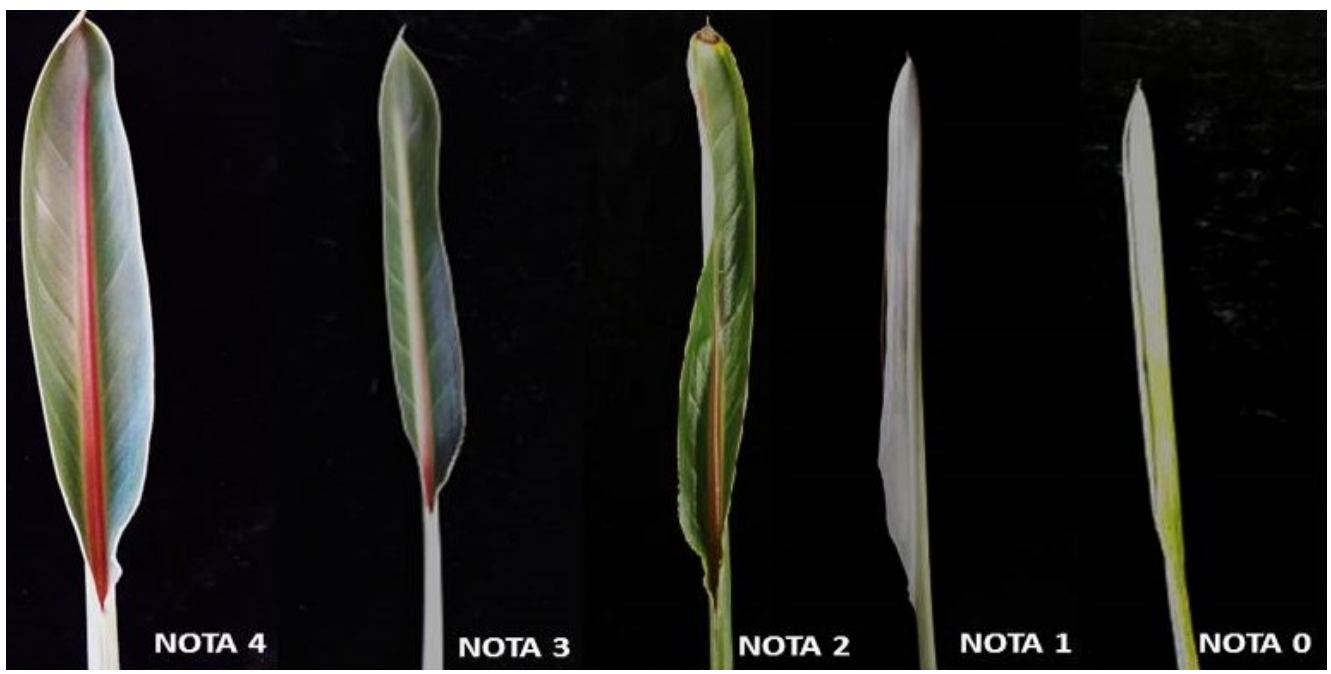

Figura 2. Critério de classificação por notas para avaliação da longevidade pó-colheita das folhas de Strelitzia juncea. Londrina/PR, 2018. Grading criteria for grades to assess postharvest longevity of Strelitzia juncea leaves. Londrina/PR, 2018.

Fonte: Autoria própria. Own authorship.

As notas obtidas foram analisadas utilizando o teste não paramétrico de Kruskal-Wallis a 5\% de significância. Para comparar os tratamentos ao longo do tempo, foi realizada uma estimativa análoga ao cálculo de área abaixo da curva de progresso da doença (AACPD) ajustado de Campbell e Madden (1990). Para estimar a área de decréscimo na qualidade das folhas em relação ao tempo de avaliação foi utilizada a seguinte equação:

$$
\mathrm{AACDQ}=\Sigma\left[\left(y_{i}+y_{i+1}\right) / 2\right]^{*}\left[t_{i+1}-t_{i}\right],
$$

em que, AACDQ: área abaixo da curva de decréscimo da qualidade das folhas; y: valores da escala de nota para avaliação da aparência foliar; e t: tempo de avaliação em dias.

Concomitantemente, foi feito o acompanhamento da perda de água nos tecidos foliares através da determinação de sua massa fresca em balança semianalítica, com precisão de $\pm 0,01 \mathrm{~g}$. 
Os dados foram coletados a cada três dias, durante o período de armazenamento de 39 dias, e os resultados expressos em porcentagem de perda de água (\%).

O delineamento experimental utilizado foi inteiramente casualizado, com cinco repetições por tratamento (dose), totalizando 6 doses de FishFertil ${ }^{\circledR}$, onde cada folha representa uma repetição, totalizando 30 folhas por espécie. Os dados foram submetidos ao teste de normalidade e homogeneidade de variância e quando aceitos os pressupostos, foi realizada a análise de variância a $5 \%$ de significância.

Para a análise das notas, os resultados foram submetidos ao teste de Kruskal-Wallis a 5\% de significância e as espécies foram comparadas utilizando o teste t de Student. Na avaliação dos dados de perda de massa, os modelos de regressão foram ajustados para modelo linear e os valores correspondentes ao coeficiente angular $(\beta)$ e linear $(\alpha)$ foram adotados, segundo a análise de variância, conforme o nível de significância. As treze avaliações ao longo do tempo também foram consideradas como fator, exceto para AACPDQ, por outro lado, a espécie não foi considerada como fator. Se significativo, foram realizadas regressões de tempo dentro de dose separadamente por espécie. Para o ajuste da dose de máxima eficiência do produto, foi adotado o modelo com ajuste quadrático, utilizando os valores de perda de peso da última avaliação (39 dias). Em todas as variáveis os dados foram processados utilizando o software $\mathrm{R}$ (R CORE TEAM, 2019).

\section{RESULTADOS E DISCUSSÃO}

As espécies $S$. reginae e $S$. juncea apresentaram os primeiros sinais de deterioração 15 dias após o início do experimento, quando a perda de peso estava em torno de 9 a $10 \%$ do total da massa fresca. Após as plantas serem colhidas, os processos de consumo das reservas energéticas ocorrem em maior intensidade e os processos de senescência são acelerados, ocasionando problemas para sua comercialização (TSEGAW et al., 2011).

A perda de massa em produtos hortícolas consiste na redução do peso fresco do produto ao longo do tempo e está diretamente relacionada à movimentação de água no produto após a colheita, determinadas pela perda de água por transpiração. Esse intenso processo de transpiração ocasiona um déficit e o produto perde água para o ambiente iniciando um estresse hídrico que tem por características a perda de turgidez e a redução do peso fresco (OLIVEIRA et al., 2017; SANCHES et al., 2017).

De acordo com a análise de regressão para ajuste das curvas comparando os tratamentos, os dados foram significativos para modelos lineares $(\mathrm{Y}=\mathrm{ax}+\mathrm{b})$. Na comparação entre as curvas para a espécie $S$. reginae, foi observado que o controle (água destilada) apresentou maior perda de peso em função do tempo quando comparado com os demais tratamentos, mostrando aos 39 dias, perda de peso médio de massa fresca de $28,4 \%$, representando aumento de $11,5 \%$ em relação ao melhor tratamento $\left(40 \mathrm{mg} \mathrm{L}^{-1}\right)(16,9 \%)$. Nos demais tratamentos a perda ao final dos 39 dias foram de 22,1\% (10 mg L-1), 17,7\% $\left(20 \mathrm{mg} \mathrm{L}^{-1}\right), 18,5 \%\left(80 \mathrm{mg} \mathrm{L}^{-1}\right)$ e $17,4 \%(160$ $\mathrm{mg} \mathrm{L}^{-1}$ ) (Figura 3). 

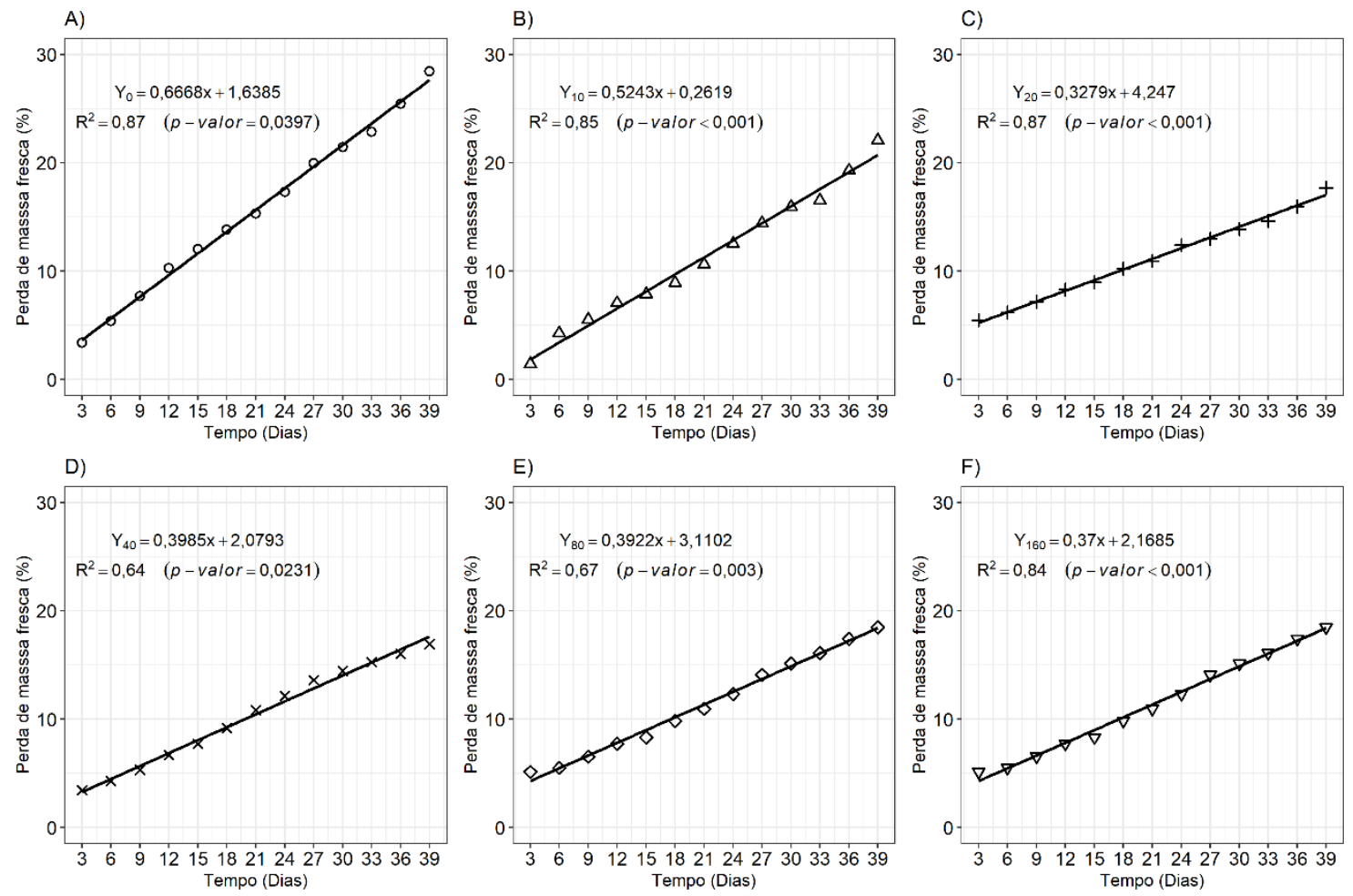

Figura 3. Porcentagem da perda de massa fresca das folhas de Strelitzia reginae em função do tempo (dias) após tratamento com seis diferentes condicionamentos (A- controle, B- $10 \mathrm{mg} \mathrm{\textrm {L } ^ { - }}$ 1, C-20 mg L ${ }^{-1}$, D- $40 \mathrm{mg} \mathrm{L}^{-1}$, E- $80 \mathrm{mg} \mathrm{L}^{-1}$ e F- $160 \mathrm{mg} \mathrm{L}^{-1}$ ). Londrina/PR, 2018. Percentage of fresh weight loss of Strelitzia reginae leaves as a function of time (days) after treatment with six different conditions(A- control, B- $10 \mathrm{mg} \mathrm{L}^{-1}, C-20 \mathrm{mg} \mathrm{L}^{-1}, D-40 \mathrm{mg} \mathrm{L}^{-1}, E-80 \mathrm{mg} \mathrm{L}^{-1}$ e F$\left.160 \mathrm{mg} \mathrm{L}^{-1}\right)$. Londrina/PR, 2018.

Fonte: Autoria própria. Own authorship.

Comportamento similar foi observado na espécie $S$. juncea, onde o controle (água destilada) apresentou maior perda de peso quando comparada com os demais tratamentos, apresentando aos 39 dias, perda de peso médio de massa fresca de $26,2 \%$, indicando $8,2 \%$ a mais em relação ao tratamento que mostrou menor perda $\left(80 \mathrm{mg} \mathrm{L}^{-1}\right)(19,0 \%)$. Os demais tratamentos apresentaram perda de 22,8\% (10 mg L $\left.\mathrm{m}^{-1}\right), 20,6 \%\left(20 \mathrm{mg} \mathrm{L}^{-1}\right), 19,0 \%\left(40 \mathrm{mg} \mathrm{L}^{-1}\right)$ e $22,7 \%\left(160 \mathrm{mg} \mathrm{L}^{-1}\right)$ (Figura 4$)$. 

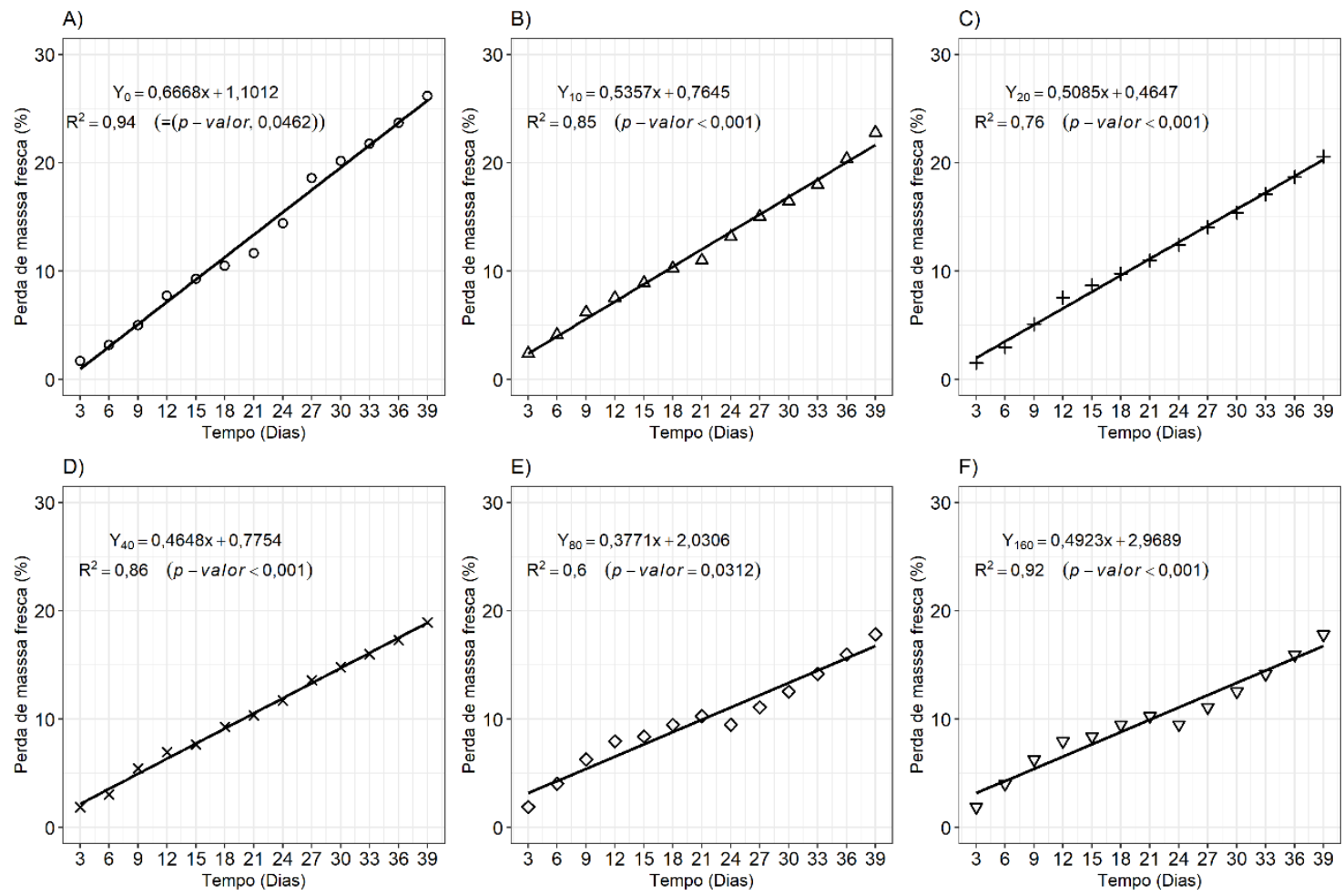

Figura 4. Porcentagem da perda de massa fresca das folhas de Strelitzia juncea em função do tempo (dias) após tratamento com seis diferentes condicionamentos (A- controle, B- $10 \mathrm{mg} \mathrm{L}^{-}$ 1, C-20 mg L-1, D- $40 \mathrm{mg} \mathrm{L}^{-1}$, E- $80 \mathrm{mg} \mathrm{L}^{-1}$ e F- $160 \mathrm{mg} \mathrm{L}^{-1}$ ). Londrina/PR, 2018. Percentage of fresh weight loss of Strelitzia juncea leaves as a function of time (days) after treatment with six different conditions (A- control, B- $10 \mathrm{mg} \mathrm{L}^{-1}, C-20 \mathrm{mg} \mathrm{L}^{-1}, D-40 \mathrm{mg} \mathrm{L}^{-1}, E-80 \mathrm{mg} \mathrm{L} \mathrm{L}^{-1}$ e F$\left.160 \mathrm{mg} \mathrm{L}^{-1}\right)$. Londrina /PR, 2018.

Fonte: Autoria própria. Own authorship.

A presença da película protetora formada pelo produto FishFertil ${ }^{\circledR}$ reduziu a perda de massa fresca das folhas durante o período de realização do experimento, em relação ao controle. A quitosana é um polissacarídeo já amplamente utilizado na pós-colheita de frutos e vegetais, este composto é largamente aplicado devido sua capacidade de formar um filme natural e permeável às trocas gasosas realizadas pelo vegetal, permitindo a redução do metabolismo da planta, fatores estes que levam a um prolongamento da vida útil (VELICKOVA et al., 2013).

Analisando todo período pós-colheita do experimento, em ambas as espécies (S. reginae e S. juncea), foi possível observar aumento da perda de massa (Figura 5). Isso se deve ao fato da água ser o principal componente das células vivas e, com o passar do tempo, esta acaba sendo eliminada no processo transpiratório e na atividade respiratória, levando ao murchamento e enrugamento, a qual pode ocorrer em poucas horas ou dias, dependendo do produto e das condições de temperatura e umidade do ar (RIBEIRO et al., 2011). Além disso, a perda de massa fresca pode promover intensos efeitos fisiológicos, interferindo na capacidade respiratória, produção de etileno e na degradação de clorofila (FRANÇA, 2011). 


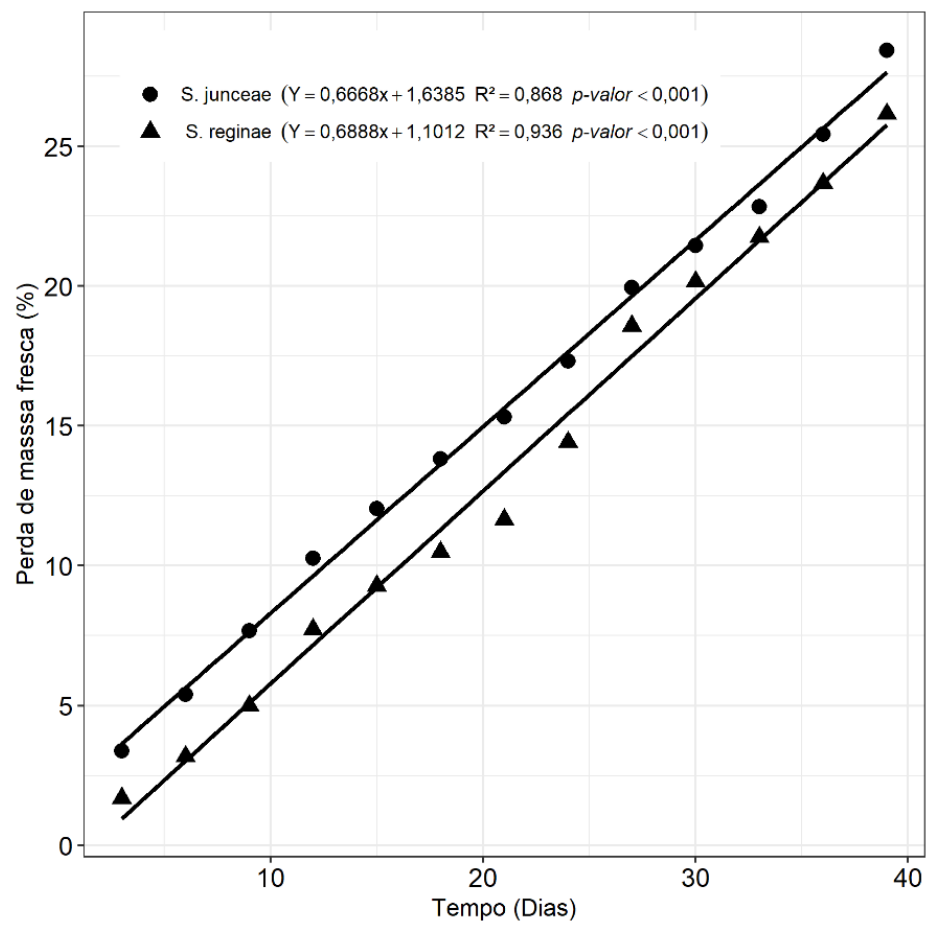

Figura 5. Porcentagem da perda de massa fresca das folhas das espécies Strelitzia reginae e Strelitzia juncea em função do tempo (dias) após tratamento com solução conservante. Londrina/PR, 2018. Percentage of fresh weight loss of leaves of the species Strelitzia reginae and Strelitzia juncea as a function of time (days) after treatment with preservative solution. Londrina / PR, 2018.

Fonte: Autoria própria. Own authorship.
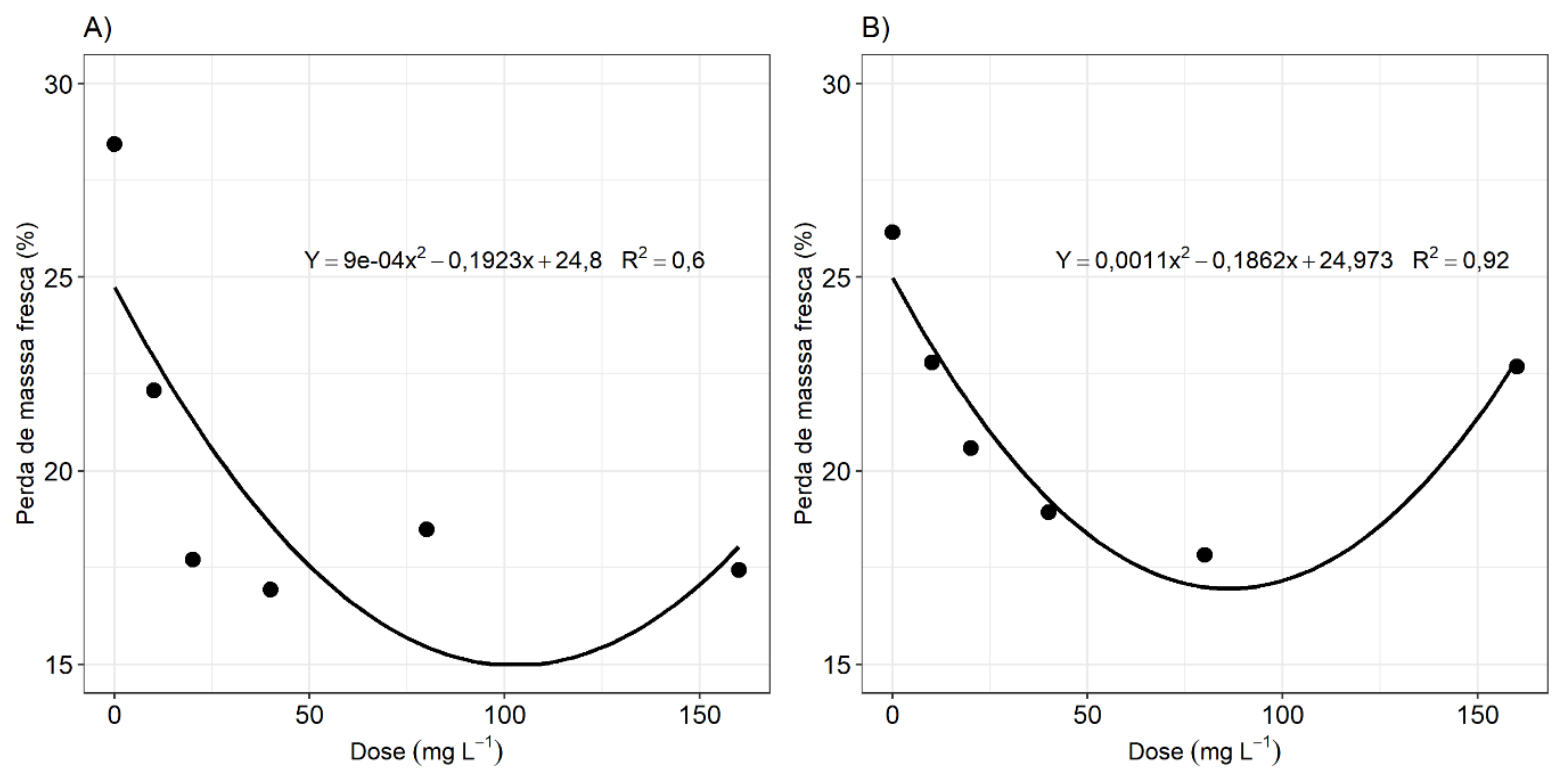

Figura 6. Porcentagem média da perda de massa fresca das folhas das espécies Strelitzia reginae (A) e Strelitzia juncea (B) em função da dose da solução conservante após 39 dias. Londrina/PR, 2018. Average percentage of fresh weight loss from the leaves of the species Strelitzia reginae (A) and Strelitzia juncea (B) as a function of the dose of the preservative solution after 39 days. Londrina / PR, 2018.

Fonte: Autoria própria. Own authorship. 
Contrapondo as diferentes doses de solução conservante a base de quitosana, verificouse que a máxima eficiência do produto, para a espécie $S$. reginae, ocorreu na dose de 106,8 $\mathrm{mg}$ $\mathrm{L}^{-1}$, na qual a perda estimada de massa fresca foi de $14,53 \%$, já para a espécie S. juncea, apresentou na dose de $84,6 \mathrm{mg} \mathrm{L}^{-1}$, cuja perda estimada de massa fresca foi de $17,09 \%$ (Fig. 6A e B).

Verificou-se maior perda de massa fresca no tratamento sem a aplicação do produto (controle), já que não há formação do recobrimento semipermeável gerado pela quitosana, a qual tem sido amplamente utilizada na proteção contra a desidratação, redução da respiração, manutenção da firmeza e coloração (ALI et al., 2011).

O efeito positivo do revestimento a base de quitosana foi evidenciado em estudos com frutos de goiaba cv. Pedro Sato (SOARES et al. 2011), e pseudofruto do cajueiro (FERREIRA et al., 2010), reduzindo a perda de massa fresca dos mesmos em relação ao controle. Oliveira et al. (2015) também verificaram maior preservação da cor natural de lichias e carambolas quando revestidas com biofilme de quitosana.

A utilização de revestimentos pode obstruir parcialmente as aberturas em que ocorrem as trocas gasosas, principalmente através da cutícula, lenticelas e também do local onde foi feita a retirada da planta mãe (PAUL; PANDEY, 2014). Além disso, possuem funções antioxidantes e antimicrobianas, além da barreira contra o oxigênio, dióxido de carbono e luz UV (WEISS et al., 2013).

Foi possível observar de acordo com a figura 6 uma tendência de sensibilidade das espécies ao produto utilizado. A modificação na atmosfera dentro de um ambiente selado, como no caso da película formada pela quitosana faz com que ocorra o consumo de $\mathrm{O}_{2}$ e liberação de $\mathrm{CO}_{2}$ (IRTWANGE, 2006). Esta atmosfera leva ao decréscimo da concentração de $\mathrm{O}_{2}$, reduzindo a velocidade de respiração e atrasa o amadurecimento e senescência de alguns produtos hortícolas (GUEVARA et al., 2003).

No entanto, altas concentrações de quitosana não impediram a redução da atividade respiratória das folhas, fato este que pode ser explicado pelo balanço de $\mathrm{O}_{2}$ e $\mathrm{CO}_{2}$, onde a concentração de $\mathrm{O}_{2}$ deve ser suficientemente baixa para retardar a respiração, porém, mais alta que a concentração crítica para o início da respiração anaeróbica. Estas condições de anaerobiose podem induzir a ao crescimento de microrganismos anaeróbicos, além do mais, o acúmulo de $\mathrm{CO}_{2}$ em níveis elevados ocasionam distúrbios fisiológicos (VILLADIEGO et al., 2005), que podem ter afetado e retardado a vida pós-colheita nas doses mais altas de quitosana.

No que se refere à análise visual (Tabela 1) das folhas, no tratamento controle, as duas espécies apresentaram grande longevidade pós-colheita, com aproximadamente 22 dias sem perder qualidade. Entretanto, o produto FishFertil ${ }^{\circledR}$ foi eficiente em prolongar a vida útil das folhas, mantendo-as até o $34^{\circ}$ dia com notas dentro do padrão comercial. 
Tabela 1. Área abaixo da curva de decréscimo da qualidade (AACDQ) e valores médios de notas atribuídas para a avaliação visual da longevidade pós-colheita de folhas das espécies Strelitzia reginae (A) e Strelitzia juncea (B), em função do tempo (dias) e da dose da solução conservante a base de quitosana (mg). Area under the quality decrease curve (AACDQ) and average score values assigned for the visual assessment of postharvest longevity of leaves of the species Strelitzia reginae (A) and Strelitzia juncea (B), as a function of time (days) and the dose of the chitosan-based preservative solution ( $\mathrm{mg}$ ).

\begin{tabular}{|c|c|c|c|c|c|c|c|c|c|c|c|c|c|c|c|c|}
\hline \multirow[t]{2}{*}{ Espécie } & \multirow{2}{*}{$\begin{array}{l}\text { Dose } \\
(\mathrm{mg} \\
\left.\mathrm{L}^{-1}\right)\end{array}$} & \multicolumn{14}{|c|}{ Tempo (Dias) } & \multirow{2}{*}{ AACDQ } \\
\hline & & C & & 4 & & 10 & & 16 & & 22 & & 28 & & 34 & 39 & \\
\hline \multirow[t]{6}{*}{ (A) } & 0 & 4,0 & $a^{*}$ & 4,0 & $\mathrm{a}$ & 3,8 & $\mathrm{a}$ & 3,8 & $\bar{a}$ & 3,8 & $\bar{a}$ & 2,0 & $\mathrm{a}$ & $2,0 \mathrm{~b}$ & $1,0 \quad b$ & 119,4 a \\
\hline & 10 & 4,0 & $\mathrm{a}$ & 4,0 & $\mathrm{a}$ & 4,0 & $\mathrm{a}$ & 3,8 & $\mathrm{a}$ & 4,0 & $\mathrm{a}$ & 3,8 & $\mathrm{a}$ & 3,6 a & $1,8 \mathrm{ab}$ & $127,8 \mathrm{ab}$ \\
\hline & 20 & 4,0 & $\mathrm{a}$ & 4,0 & $\mathrm{a}$ & 4,0 & $\mathrm{a}$ & 4,0 & $\mathrm{a}$ & 3,6 & $\mathrm{a}$ & 3,6 & $\mathrm{a}$ & 3,6 a & $2,4 \mathrm{ab}$ & $140,6 \quad b$ \\
\hline & 40 & 4,0 & $\mathrm{a}$ & 4,0 & $\mathrm{a}$ & 4,0 & $\mathrm{a}$ & 4,0 & $\mathrm{a}$ & 3,6 & $\mathrm{a}$ & 3,6 & $\mathrm{a}$ & 3,6 a & $2,4 \mathrm{ab}$ & 140,6 \\
\hline & 80 & 4,0 & $\mathrm{a}$ & 4,0 & $\mathrm{a}$ & 3,8 & $\mathrm{a}$ & 3,6 & $\mathrm{a}$ & 3,6 & $\mathrm{a}$ & 3,6 & $\mathrm{a}$ & 3,6 a & $2,4 a b$ & $132,4 \mathrm{ab}$ \\
\hline & 160 & 4,0 & $\mathrm{a}$ & 4,0 & $\mathrm{a}$ & 4,0 & $\mathrm{a}$ & 4,0 & $\mathrm{a}$ & 3,8 & $\mathrm{a}$ & 3,6 & $\mathrm{a}$ & 3,6 a & $2,2 \mathrm{ab}$ & $138,2 \mathrm{ab}$ \\
\hline \multirow[t]{6}{*}{ (B) } & 0 & 4,0 & $\mathrm{a}$ & 4,0 & $\mathrm{a}$ & 4,0 & $\mathrm{a}$ & 4,0 & $\mathrm{a}$ & 3,8 & $\mathrm{a}$ & 3,8 & $\mathrm{a}$ & $3,0 \quad \mathrm{a}$ & $2,0 \mathrm{ab}$ & $137,2 \quad \mathrm{a}$ \\
\hline & 10 & 4,0 & $\mathrm{a}$ & 4,0 & $\mathrm{a}$ & 4,0 & $\mathrm{a}$ & 4,0 & $\mathrm{a}$ & 3,6 & $\mathrm{a}$ & 3,6 & $\mathrm{a}$ & 3,6 a & $3,0 \mathrm{a}$ & 140,6 a \\
\hline & 20 & 4,0 & $\mathrm{a}$ & 4,0 & $\mathrm{a}$ & 4,0 & $\mathrm{a}$ & 3,8 & $\mathrm{a}$ & 3,8 & $\mathrm{a}$ & 3,8 & $\mathrm{a}$ & 3,6 a & 3,0 a & 140,6 a \\
\hline & 40 & 4,0 & $\mathrm{a}$ & 4,0 & $\mathrm{a}$ & 4,0 & $\mathrm{a}$ & 4,0 & $\mathrm{a}$ & 3,6 & $\mathrm{a}$ & 3,6 & $\mathrm{a}$ & 3,6 a & 3,0 a & 140,6 a \\
\hline & 80 & 4,0 & $\mathrm{a}$ & 4,0 & $\mathrm{a}$ & 4,0 & $\mathrm{a}$ & 4,0 & $\mathrm{a}$ & 3,8 & $\mathrm{a}$ & 3,6 & $\mathrm{a}$ & 3,6 a & $3,0 \mathrm{a}$ & $142,2 \mathrm{a}$ \\
\hline & 160 & 4,0 & $\mathrm{a}$ & 4,0 & $\mathrm{a}$ & 4,0 & $\mathrm{a}$ & 4,0 & $\mathrm{a}$ & 3,4 & $\mathrm{a}$ & 3,6 & $\mathrm{a}$ & 3,6 a & $2,6 a b$ & 139,2 a \\
\hline
\end{tabular}

*Médias seguidas de letras minúscula distintas na coluna indicam diferença significativa entre as médias. O teste não paramétrico utilizado foi de Kruskal-Wallis. * Means followed by distinct lower case letters in the column indicate a significant difference between the means. The non-parametric test used was Kruskal-Wallis.

Fonte: Autoria própria. Own authorship.

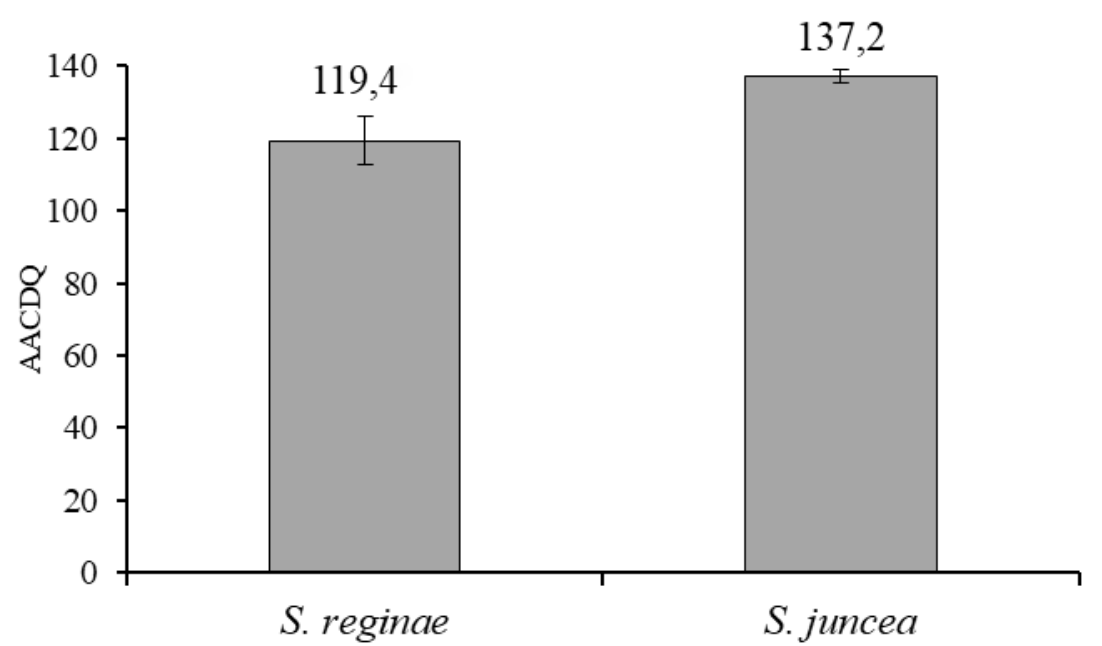

Figura 7. Área abaixo da curva de decréscimo da qualidade (AACDQ) em função da espécie de Strelitzia. Londrina/PR, 2018. Area below the quality decrease curve (AACDQ) as a function of the Strelitzia species. Londrina / PR, 2018.

Fonte: Autoria própria. Own authorship.

Analisando a AACDQ (Area abaixo da curva de descréscimo de qualidade), a espécie $S$. juncea apresentou uma prolongação da qualidade pós-colheita quando comparada a espécie $S$. 
reginae, uma vez que os valores apresentados na Figura 7 para a espécie $S$. reginae são inferiores aos indicados para $S$. juncea.

O produto comercial a base de quitosana $\left(\right.$ FishFertil $\left.^{\circledR}\right)$, retardou a perda de massa fresca das folhas de $S$. juncea e $S$. reginae e as mantiveram até aproximadamente o $34^{\circ}$ dia com notas dentro do padrão comercial. Entretanto, não foi possível determinar a dose de máxima eficiência do produto para o tratamento de folhas das duas espécies de Strelitzia.

\section{CONCLUSÃO}

O produto comercial a base de quitosana $\left(\right.$ FishFertil $\left.{ }^{\circledR}\right)$, retardou a perda de massa fresca das folhas de $S$. juncea e $S$. reginae e as mantiveram até aproximadamente o $34^{\circ}$ dia com notas dentro do padrão comercial. Entretanto, não foi possível determinar a dose de máxima eficiência do produto para o tratamento de folhas das duas espécies de Strelitzia.

\section{AGRADECIMENTOS}

Os autores agradecem a Coordenação de Aperfeiçoamento de Pessoal de Nível Superior (CAPES) pela bolsa cedida ao primeiro autor ( $\left.n^{\circ} 88882.448347 / 2019-01\right)$ e ao Conselho Nacional de Desenvolvimento Científico e Tecnológico (CNPq) pelo apoio financeiro ao terceiro autor ( $\left.{ }^{\circ} 141699 / 2020-5\right)$ e ao último autor $\left(n^{\circ} 301684 / 2017-0\right)$ e a Universidade Estadual de Londrina (UEL).

\section{REFERÊNCIAS BIBLIOGRÁFICAS}

ALI, A.; MUHAMMAD, M. T. M.; SIJAM, K.; SIDDIQUI, Y. Effect of chitosan coatings on the physicochemical characteristics of 'Eksotika II' papaya (Carica papaya L.) fruit during cold storage. Food Chemistry, Semenyih, v. 124, n. 2, p.620-626, 2011. Disponível em: http://dx.doi.org/10.1016/j.foodchem.2010.06.085. Acesso em: 15 fev. 2020.

AUSTRALIA. Redlands Nursery. Homelovers. Plants that perfom for you. 2019. Disponível em: http:/www.menbers.ozemail.com.au/ redny/infonotes/strelitziareginae.html. Acesso em: 08 ago. 2019.

CAMPBELL, C. L.; MADDEN, L. V. Introduction to plant disease epidemiology. New York, NY: Wiley. 1990. 532p.

DIAS-TAGLiACOZZO, G. M.; CASTRO, C. E. F. 2005. Manutenção da qualidade póscolheita de Zingiber spectabile Griff. Horticultura Brasileira, Brasília, v. 23, n. 2, p.563, 2005.

DIAS-TAGLiACOZZO, G. M.; MOSCA, J. L. Pós-colheita de flores e Folhagem: Manutenção da qualidade. Revista Brasileira de Horticultura Ornamental, Fortaleza, v. 13, n. 1 , p.209219, 2007. 
FERREIRA, A. P. S.; ISEPON, J. S.; MOREIRA, E. R.; ATÍlIO, L. B.; BOLIANI, A. C. Qualidade pós-colheita do pseudofruto do cajueiro sob ação de quitosana e refrigeração. Cultura Agronômica, Ilha Solteira, v. 19, n. 1, p.64-70, 2010.

FRANÇA, C.F.M. Conservação e qualidade pós-colheita em duas Variedades de alface submetidas ao hidroresfriamento. 2011. 44 f. Dissertação (Mestrado em Fitotecnia) Universidade Federal de Viçosa, Minas Gerais, 2011.

GUEVARA, J. C.; YAHIA, E. M.; LA FUENTE, E. B. ; BISERKA, S. P. Effects of elevated concentrations of $\mathrm{CO}_{2}$ in modified atmosphere packaging on the quality of prickly pear cactus stems (Opuntia spp.). Postharvest Biology and Technology, Cidade do México, v. 29, n. 2, p.167-176, 2003. Disponível em: http://dx.doi.org/10.1016/S0925-5214(03)00021-8. Acesso em: 17 jan. 2020.

GUIMARÃES, A. A. Armazenamento refrigerado de Heliconia bihai associado á atmosfera modificada. 159f. Tese (Doutorado em Fitotecnia) - Universidade Federal de Viçosa, Minas Gerais, 2008.

IBRAFLOR. Dados gerais do setor condensado. Disponível em: http://www.ibraflor.com. Acesso em: 19 out. 2020.

IRTWANGE, S.V. Application of modified atmosphere packaging and related technology in postharvest handling of fresh fruit and vegetables. Agricultural Engineering International, Makurdi, v. 8, n. 4, p.1-13, 2006.

LORENZI, H., SOUZA, H, M. Plantas ornamentais do Brasil: arbustivas, herbáceas e trepadeiras. Nova Odessa: Instituto Plantarum, 2008. 1088p.

MACNISH, A. J.; REID, M. S.; MARRERO, A.; JIANG, C. Z. Improving the postharvest performance of bird-of-paradise flowers. Acta Horticulturae, v. 877, p. 1763-1769, 2009. Disponível em: https://doi.org/10.17660/ActaHortic.2010.877.242. Acesso em: 19 dez. 2020.

NEVES, M. F.; PINTO, M. J. A. Mapeamento e quantificação da cadeia de flores e plantas ornamentais do Brasil. São Paulo: OCESP, 2015. 122p.

OLIVEIRA, O. M.; MENEZES, K. R. P.; SANTOS, G. C. S.; SANCHES, A. G.; CORDEIRO, C. A. M. Embalagem e tratamento hidrotérmico na manutenção da qualidade pós-colheita de jambu. Revista de Agricultura Neotropical, Cassilândia, v. 4, n. 3, p.41-49, 2017. Disponível em: https://doi.org/10.32404/rean.v4i3.1611. Acesso em: 04 dez. 2020.

OLIVEIRA, T. A.; AROUCHA, E. M. M.; LEITE, R. H. L.; FERREIRA, R. M. A., SANTOS, F. K. G. Conservação pós-colheita de carambola sob refrigeração com recobrimento de biofilme de gelatina e PVC. Revista Verde, Pombal, v. 10, n. 4, p.59-66. 2015. Disponível em: https://doi.org/10.18378/rvads.v10i4.3653. Acesso em: 10 jan. 2020.

PAUL, V.; PANDEY, R. Role of internal atmosphere on fruit ripening and storability -a review. Journal Food Science and Technology, v. 51, n. 7, p.1223-1250, 2014. Disponível em: https://doi.org/10.1007/s13197-011-0583-x. Acesso em: 17 jan. 2020. 
PIVETTA, K. F. L.; BARBOSA, J. G.; ARAÚJO, E. F. Propagação de palmeiras e Strelitzia. In: Barbosa, J. G., Lopes, L. C. (Ed). Propagação de Plantas Ornamentais. v. 1. Viçosa: UFV, 2007. p. 43-70

R CORE TEAM R. A language and environment for statistical computing. 2019. R Foundation for Statistical Computing. Vienna. Disponível em: http://www.r-project.org. Acesso em: 18 jun.2019.

RIBEIRO, W. S.; BARBOSA, J. A.; COSTA, L. C.; BRUNO, R. L. A.; ALMEIDA, E. I. B.; SILVA, K. R. G.; BRAGA JÚNIOR, J. M.; BEZERRA, A. K. D. Conservação e fisiologia póscolheita de folhas de Capuchinha (Tropaeolum majus L.). Revista Brasileira de Plantas Medicinais, Botucatu, v. 13, p.598-605, 2011.

SANCHES, A. G.; SILVA, M. B.; COSTA, M. J. M.; MOREIRA, E. G. S.; COSME, S. S.; CORDEIRO, C. A. M. Avaliação da qualidade de alfaces minimamente processadas cultivadas em sistema hidropônico. Revista Trópica: Ciências Agrárias e Biológicas, Chapadinha, v. 10, n. 1, p.19-31, 2017.

SOARES, N. F. F.; SILVA, D. F. P.; CAMILlOTO, G. P.; OLIVEIRA, C. P.; PINHEIRO, N. M.; MEDEIROS, E. A. A. Uso de revestimento comestível e conservação pós-colheita de goiaba. Revista Brasileira de Fruticultura, Jaboticabal, v. 33, n. 1, p.281-289, 2011. Disponível em: https://doi.org/10.1590/S0100-29452011000500035. Acesso em: 14 fev. 2020

TSEGAW, T.; TILAHUN, S.; HUMPHRIES, G. Influence of pulsing biocides and preservative solution treatment on the vase life of cut rose (Rosa hybrida L.) varieties. Ethiopian Journal of Applied Sciences and Technology, Jimma, v. 2, n. 2, p.1-18, 2011. Disponível em: http://dx.doi.org/10.4314/star.v4i3.12. Acesso em: 07 fev. 2020.

VELICKOVA, E.; WINKELHAUSEN, E.; KUZMANOVA, S.; ALVES, V.D.; MOLDÃOMARTINS, M. Impact of chitosan-beeswax edible coatings on the quality of fresh strawberries (Fragaria ananassa cv Camarosa) under commercial storage conditions. Food Science and Technology, Campinas, v. 52, n. 2, p.80-92, 2013. Disponível em: https://doi.org/10.1016/j.lwt.2013.02.004. Acesso em: 15 fev. 2020.

VIEIRA, M. R. S.; SIMÕES, A. N.; NUNES, G. H. S.; SOUSA, P. A. Effect of low temperature storage on conservation varieties of Chrysanthemum cutting. Journal of Stored Products and Postharvest Research, Edimburgo, v. 4, n. 4, p.51-54, 2013. Disponível em: https://doi.org/10.5897/JSPPR2013.0155. Acesso em: 10 fev. 2020.

VIEIRA, M. R. S.; LIMA, G. P. P.; MEDEIROS, D. C.; SOUZA, A. V.; OLIVEIRA, E. C. A. Genus: Strelitzia. Journal of Horticulture and Forestry, Ongole, v. 4, n. 11, p.178-180, 2012. https://doi.org/10.5897/JHF12.025. Acesso em: 23 jan. 2020.

VILLADIEGO, A.M.D.; SOARES, N.F.F.; ANDRADE, N.J.; PUSCHMANN, R.; MINIM, V.P.R.; CRUZ, R. Filmes e revestimentos comestíveis na conservação de produtos alimentícios. Revista Ceres, Viçosa, v. 52, n. 300, p.221-244, 2005.

WEISS, E. A.; OLDHAM, G.; LIN, M.; FOSTER, T.; QUINN, J. V. Water is a safe and effective alternative to sterile normal saline for wound irrigation prior to suturing: a prospective, double-blind, randomised, controlled clinical trial. British Medical Journal Open, Londres, 
v. 3, e001504, 2013. Disponível em: https://doi.org/10.1136/bmjopen-2012-001504. Acesso em: 11 fev. 2020. 